\title{
VEGF promotes stemness
}

In addition to inducing angiogenesis, vascular endothelial growth factor (VEGF) signalling may directly affect tumour cells. Using a mouse model of chemically induced skin tumours, Cédric Blanpain and colleagues have investigated the role of VEGF in cancer stem cells (CSCs).

Tumour epithelial cells (TECs) that express CD34 were previously identified as CSCs in DMBA- and TPA-induced skin papillomas in mice. The authors used immunostaining of several cutaneous CSC, TEC and endothelial cell markers to show that most CSCs in DMBA- and TPA-induced skin papillomas co-expressed TEC markers and that they were near endothelial cells, indicating that these CSCs may reside in a vascular niche. The treatment of the skin papillomas with an antibody that inhibits VEGF receptor 2 (VEGFR2) not only reduced angiogenesis as previously reported, but also reduced the proliferation and number of CD34+ CSCs.

To investigate a possible direct effect of VEGF on CSCs, the authors first showed that $\mathrm{CD} 34^{+} \mathrm{CSC}$ express higher levels of Vegfa than CD34- TECs. Conditional deletion of Vegfa in mice with established skin papillomas led to the disappearance of most of the tumours.
This was associated with reduced angiogenesis, but it also correlated with a reduction in the number of $\mathrm{CD} 34^{+} \mathrm{CSCs}$ and a reduction in the proportion of symmetric progenitor self-renewing cell divisions (as opposed to asymmetric differentiating divisions). Furthermore, overexpression of VEGFA in TECs following skin papilloma formation increased tumour growth, angiogenesis, the number of $\mathrm{CD} 34^{+} \mathrm{CSCs}$ and symmetric cell divisions.

Analysis of the expression of various VEGF receptors by quantitative reverse transcription PCR and immunostaining in $\mathrm{CD}_{34}{ }^{+} \mathrm{CSC}$ revealed that neuropilin 1 (NRP1), which is a VEGF co-receptor, was most highly expressed. In mice lacking epidermal Nrp1, DMBA and TPA did not induce any papillomas after 25 weeks; by contrast, all control mice developed papillomas. Simultaneous deletion of Nrp1 and overexpression of VEGFA in TECs blocked the ability of VEGFA to stimulate tumour growth, expansion of CD $34^{+}$ CSCs and symmetric cell division, indicating that NRP1 mediates the autocrine effects of VEGFA on CSCs. In addition, in vitro proliferation of CD34+ TECs, but not CD34- TECs,

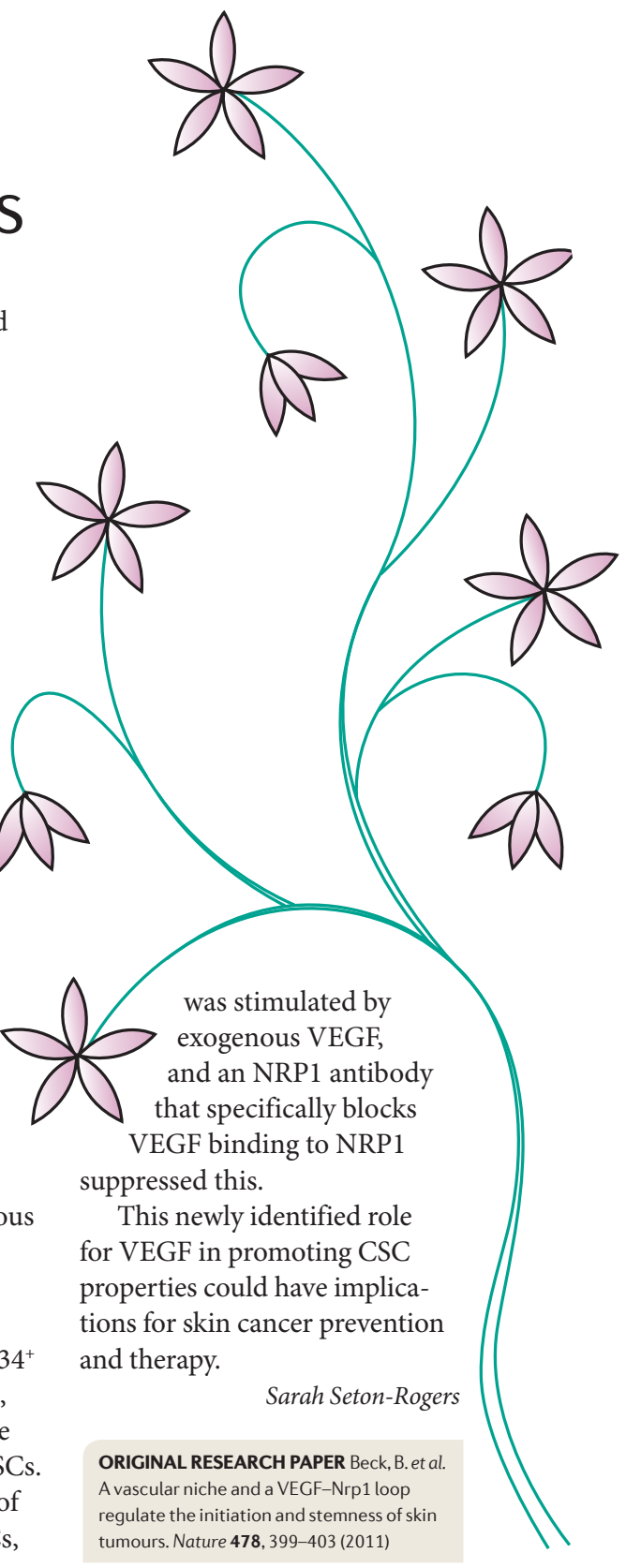

